\title{
Bound State Excitation, Nucleon Decay Experiments and Models of Wavefunction Collapse
}

\author{
Philip Pearle \\ Hamilton College \\ Clinton, New York, 13923, U.S.A. \\ and \\ Euan Squires \\ Department of Mathematical Sciences \\ University of Durham \\ Durham City, DH1 $3 L E$, England
}

August, 1993

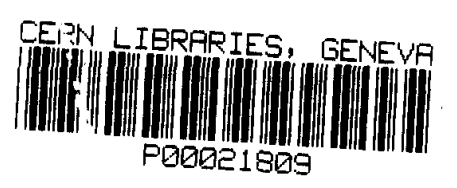

\begin{abstract}
We discuss bound-state excitation in models where wavefunction collapse is a physical process. Consequences of a feasible photon counting experiment are spelled out, were an upper limit to be found on the rate of anomalous photon emission from solid matter that is better than one photon/sec.cc.. It is also pointed out that experiments already performed, which give an upper bound on the rate of nucleon decay, can have significant consequences for such models. We illustrate by showing how these experimental results could be considered to rule out the original version of the collapse models, and support the continuous collapse versions in which excitation is considerably reduced, especially if the rate of the collapse process is proportional to the mass, indicative of a gravitational mechanism for collapse.
\end{abstract}

PCS: 03.65

There now exists a class of models in which the evolution of the wavefunction is altered from that of standard quantum theory in order to implement explicit collapse of the wavefunction. ${ }^{1} \mathrm{John} \mathrm{Bell}^{2}$ publicized such models as superior to standard quantum theory in that they allow a realistic version of quantum theory in which measurements are properly described by the equations, and no further interpretation is required.

Although the explicit collapse process makes the behaviour of a macroscopic object radically different to that given by orthodox quantum theory, the models are so constructed that there is approximate agreement for microscopic systems containing small numbers of particles. Nevertheless, there are small differences which are in principle detectable. In particular, the narrowing of wavepackets by the collapse leads to an increase of energy, in violation of the conservation rule. ${ }^{1,3}$ The energy of a collection of bound particles grows due to an increase in the centre-of-mass kinetic energy and also due to transitions to excited states. (Whether we should regard this as being a transfer of energy, e.g., from the stochastic field whose interaction with the particles causes the collapse in the CSL model discussed below, or a genuine violation of energy conservation does not affect the present discussion). 

Since we are dealing with a fundamental process it is reasonable to assume that it applies to all the basic constituents of matter, in particular, to electrons and quarks. Thus, for example, the electrons in atoms will become excited, and matter should spontaneously radiate photons. Below we shall review and expand the previous discussion of this effect.

Here we shall also extend this idea to the quarks in nucleons. Thus, for example, the quarks in protons will become excited and protons should spontaneously radiate pions. We point out that the extraordinarily long lower limit on the nucleon lifetime has the potential to put severe restrictions on the parameters of collapse models, and also on the form such models should take.

To illustrate, we present the case that the spontaneous localisation (SL) model of Ghirardi, Rimini and Weber ${ }^{3}$ (GRW) can become untenable under the hypothesis that the parameters which characterize the model are the same for electrons and quarks. We further argue that the continuous spontaneous localization (CSL) model ${ }^{4}$ is rescued from marginal tenability only if the rate of wavefunction collapse is proportional to the mass of the particle involved, as in the modification of Diosi's model ${ }^{5}$ by Ghirardi, Grassi and Rimini ${ }^{6}$, thereby supporting the belief that wavefunction reduction is related to gravity ${ }^{7}$.

Two parameters were introduced by GRW to characterise their SL model. One, $a$, essentially gives the spatial spread of the collapsed wavefunction of a single particle. The other, $T$, is the average time between successive collapses of a single particle wavefunction. If we consider a solid macroscopic "pointer" composed of $N$ particles responsive to the collapse mechanism (there could of course be other, unresponsive, constituents), and suppose that its wavefunction is initially in a superposition of two spatially nonoverlapping states (separation greater than $a$ ), it will collapse to one of these states in a time

$$
\tau_{S L} \approx \frac{T}{N}
$$

With the GRW value, $T=10^{16} \mathrm{sec} \approx 300$ million years, the state of a macroscopic pointer containing, say, $N \sim 10^{23}$ particles will collapse in a time of the order of $10^{-7} \mathrm{sec}$, a value substantially less than human perception times, which are greater than about $10^{-2}$ sec.

In order that the pointer collapses at this rate whenever the superposition involves observably different states, the value of $a$ must be smaller than separations that are distinguishable by visible light, i.e., $\sim 10^{-4} \mathrm{~cm}$. On the other hand it must not be too small, otherwise the rate of energy increase, which is in principle detectable, would become unacceptably large. GRW chose the value $a=10^{-5} \mathrm{~cm}$.

In SL the collapse-induced rate of increase of the average energy for a system of $N$ equal-mass particles, with each particle possessing identical values for $a$ and $T$, is given exactly by

$$
\frac{d \bar{H}}{d t}=\frac{3 \hbar^{2}}{4 m a^{2}} \frac{N}{T}
$$

for any state of the system. The increase of the c.m. energy is given by the same expression without the factor $N$. If the particles belong to a bound state whose 
spatial extension is much less than $a$, then the probability/sec of a transition from this initial state $\left|\psi_{0}\right\rangle$, in which the bound system is in its ground state, to an orthogonal state $|\phi\rangle$, in which the this system is excited, is given, to leading order in $a^{-2}$, by

$$
\dot{P}(\phi)=\frac{1}{T} \sum_{k=1}^{N} \frac{|<\phi| \mathbf{x}_{k}\left|\psi_{0}>\right|^{2}}{2 a^{2}}
$$

where $\mathbf{x}_{k}$ is the position operator of the $k^{\text {th }}$ particle.

If $a$ is chosen much smaller than GRW's value then the energy non-conservation predicted by the model should certainly be detectable. Suppose, for example, the effect happens to electrons. Then eq.(3) predicts that atoms in a typical solid will radiate about 1 photon per cubic centimetre per second (call this rate $\left.\Gamma_{G R W}\right){ }^{8}$ These photons would be mainly due to transitions between the first excited state and the ground state. Surprisingly, as far as we are aware, there does not exist an experimental upper limit on the "anomalous" flux of photons from matter. Even independently of its relevance to the present discussion, there is an experiment here which is surely worth doing.

An experimental limit can be found when eq.(3) is applied to calculate the expected flux of Lyman- $\alpha$ ultraviolet photons emitted by intergalactic hydrogen ${ }^{9}$. It turns out that the GRW values give a flux that is about $2.10^{7}$ times smaller than that which is observed. Hence, without trying to improve the limit by taking into account other possible sources of this flux, we can certainly say that $\left(T a^{2}\right)^{-1}$ has an experimental upper bound given by

$$
\left(T a^{2}\right)_{e x p}^{-1}<2.10^{7}\left(T a^{2}\right)_{G R W}^{-1}=20 \mathrm{~cm}^{-2} \sec ^{-1} .
$$

Were terrestial experiments to give a flux upper limit significantly better than $\Gamma_{G R W}$, this could be taken as ruling out the SL model. As we shall see below, nucleon lifetime experiments can be construed as giving a much more stringent bound on $\left(T a^{2}\right)^{-1}$ (see eq.(14)), which indeed is not compatible with the GRW values.

The continuous spontaneous localisation (CSL) model of wavefunction collapse represents an improvement on the SL model, in that it describes a continuous process by a modified Schrödinger equation. Moreover, it preserves the symmetry of the wavefunction for identical particles, which the SL model fails to do. In CSL the reduction time for a pointer becomes

$$
\tau_{C S L}=\frac{T}{N D a^{3}},
$$

where $D$ is the particle-number density. This differs from the SL result in eq.(1) by the factor corresponding to the inverse of the average number of particles in the "collapse volume", which is typically of the order of $10^{9}$. Thus, with the GRW values, the collapse time of a pointer containing $10^{23}$ particles decreases from around $10^{-7}$ to $10^{-16}$ sec.

Remarkably, the rate of energy increase is again given exactly by eq. (2). (This is because only the $j=k$ terms in eq.(6) below contribute). 
The CSL expression for the probability/sec of excitation of a bound state (localised well within the distance $a$ ), which replaces eq.(3), has not been given previously, so we sketch the derivation here. The equation for the evolution of the density matrix, $\rho(t)$, in CSL is ${ }^{4}$

$$
\begin{aligned}
\frac{\partial<x|\rho| x^{\prime}>}{\partial t} & =-i<x|[H, \rho]| x^{\prime}> \\
& -\frac{1}{2 T} \sum_{j=1}^{N} \sum_{k=1}^{N}\left[\Phi\left(\mathbf{x}_{j}-\mathbf{x}_{k}\right)+\Phi\left(\mathbf{x}_{j}^{\prime}-\mathbf{x}_{k}^{\prime}\right)-2 \Phi\left(\mathbf{x}_{j}-\mathbf{x}_{k}^{\prime}\right)\right]<x|\rho| x^{\prime}>(6)
\end{aligned}
$$

where $|x\rangle=\left|\mathbf{x}_{1}, \mathbf{x}_{2}, \ldots\right\rangle$ is the $N$-particle position eigenstate, and $\Phi(\mathbf{z})=\exp \left[\frac{-z^{2}}{4 a^{2}}\right]$, although any other similarly behaved function would do equally well. For comparison purposes the corresponding equation in SL (which was used in obtaining the results in eqs. (1-3) ) is

$$
\frac{\partial<x|\rho| x^{\prime}>}{\partial t}=-i<x|[H, \rho]| x^{\prime}>-\frac{1}{T} \sum_{k=1}^{N}\left[1-\Phi\left(\mathbf{x}_{k}-\mathbf{x}_{k}^{\prime}\right)\right]\left\langle x|\rho| x^{\prime}>\right.
$$

Since $\dot{P}(\phi)=\frac{\partial\langle\phi|\rho| \phi\rangle}{\partial t}$, we multiply eq.(6) by $\langle\phi \mid x\rangle\left\langle x^{\prime} \mid \phi\right\rangle$ and integrate over $x$ and $x^{\prime}$. Initially $\rho=\rho(0)=\left|\psi_{0}><\psi_{0}\right|$, so all terms except those involving $\Phi\left(\mathbf{x}_{j}-\mathbf{x}_{k}^{\prime}\right)$ vanish because of the orthogonality of $|\phi\rangle$ and $|\psi\rangle$. Then if we expand this function as $1-\left(x_{j}^{2}+x_{k}^{\prime 2}-2 \mathbf{x}_{j} \cdot \mathbf{x}_{k}^{\prime}\right) / 4 a^{2}$ we obtain

$$
\begin{aligned}
\left.\dot{P}(\phi)\right|_{t=0} & =\frac{1}{2 T a^{2}} \sum_{j=1}^{N} \sum_{k=1}^{N}\left\langle\phi\left|\mathbf{x}_{j}\right| \psi_{0}\right\rangle .\left\langle\psi_{0}\left|\mathbf{x}_{k}\right| \phi\right\rangle \\
& =\frac{N^{2}}{2 T a^{2}}|<\phi| \mathbf{Q}\left|\psi_{0}>\right|^{2},
\end{aligned}
$$

where $\mathbf{Q}$ is the c.m. position operator of the particles considered, $\mathbf{Q}=N^{-1} \sum_{j} \mathbf{x}_{j}$.

If we suppose that only electrons are affected by the collapse process, then eq.(8) gives essentially $\Gamma_{G R W}$, the same rate of photoemission as eq.(3). However, the situation can change if we postulate that the process happens for both electrons and nucleons (here we regard these as elementary particles and ignore their quark structure). In this case there is of course freedom to introduce different parameters, $a$ and $T$, for different particles. Also, recalling that the CSL process is caused by a fluctuating field in space-time, we could allow different particles to be governed by different such fields. Then the double sum in eq.(6), for example, would be replaced by a sum of such terms, one for each type of particle. A more economical proposal is to suppose that there is just one type of fluctuating field. Then the double sum is over all particles of whatever type. Moreover, the $a$ parameter must then be common, since $\left(a_{1}^{-1} \mathbf{x}_{1}-a_{2}^{-1} \mathbf{x}_{2}\right)$ is not Galilean invariant unless $a_{1}=a_{2}$.

There is however still the freedom to allow the coupling to the fluctuating field to depend on the particle type. The effect of this is to introduce a factor $\mu_{j} \mu_{k}$ 
into the double sums of eqs (6) and (8a), where $\mu_{j}$ is a dimensionless constant characterising the coupling of particle $j$ to the fluctuating field. We now note that one particular choice of the $\mu_{j}$ greatly reduces the rate of particle excitation in CSL, namely, $\mu_{j}=m_{j} / m_{0}$, where $m_{j}$ is the mass of the $j^{t h}$ particle and $m_{0}$ is a constant. Then, analogous to eq.(8), we obtain

$$
\begin{aligned}
\left.\dot{P}(\phi)\right|_{t=0} & =\frac{M^{2}}{2 T a^{2} m_{0}^{2}}|<\phi| \mathbf{Q}\left|\psi_{0}>\right|^{2} \\
& =0
\end{aligned}
$$

where now $\mathbf{Q}$ is the c.m. position of all the particles, and $M$ is the total mass. The last equation $(9 \mathrm{~b})$ holds because the c.m. operator cannot excite the internal atomic states.

With this choice for the mass dependence of the couplings, the average energy increase, which is now given by

$$
\frac{d \bar{H}}{d t}=\frac{3 \hbar^{2}}{4 m_{0} a^{2}} \frac{M}{m_{0} T}
$$

is totally due to c.m. energy. For completeness we note that the pointer collapse rate, replacing eq. (5), is given by

$$
\tau_{C S L}=\frac{T m_{0}^{2}}{M D_{M} a^{3}}
$$

$M$ being the total mass of the pointer, and $D_{M}$ its mass density.

It follows from the above results that the first non-vanishing contribution to the rate of internal excitation of atoms comes from the next order in the expansion of the function $\Phi$, leading to

$$
\dot{P}(\phi)_{t=0} \sim \frac{1}{T}\left(\frac{m_{e}}{m_{0}}\right)^{2}\left(\frac{a_{0}}{a}\right)^{4}
$$

where $m_{e}$ is the electron mass and $a_{0}$ is the atomic radius.

Suppose we take $m_{0}$ to be the mass of the nucleon so that the collapse time for an isolated nucleon, $\sim T\left(\frac{m_{0}}{m}\right)^{2}$, is unchanged from the GRW value. Also unchanged is the c.m. energy increase of hydrogen gas given by eq.(2) or eq.(10) which amounts to about .001 degrees Kelvin over the age of the universe. ${ }^{1,3}$ (The collapse time for an electron is of course increased to $T\left(\frac{m_{0}}{m_{e}}\right)^{2} \approx 10^{22}$ sec.) From eq.(12) we see that the atomic excitation rate is about a factor $10^{-12}$ down from $\Gamma_{G R W}$, given by eq.(3) or eq.(8), corresponding to a rate from ordinary matter of one photon per cubic centimetre per 100,000 years. So, to summarise, if the coupling is proportional to the particle mass, there is considerable reduction of the predicted rate of bound state excitation for a given choice of the GRW parameters, $a$ and $T$.

We now turn to the problem of the collapse-induced excitation of the quarks in a nucleon. On the experimental side we certainly expect that the predicted excitation 
of the lowest $I=\frac{1}{2}$ excited state, at $1440 \mathrm{Mev}$, would be detected by the nucleon decay experiments, since the decay pion(s) is (are) in the observable energy range of these experiments. ${ }^{10}$ In a typical such experiment, a detector of a few kilotons sees about one event per day. Careful analysis of these events, and comparison with expected neutrino-induced processes, suggests that they are all compatible with being caused by neutrinos. It is concluded that the nucleon lifetime is greater than $10^{31}$ years $\approx 3.10^{38} \mathrm{sec}$ with a 90 percent confidence level.

Since collapse-induced decays would be similar to the "Grand-unified" decays that the experiments were designed to detect, it is not unreasonable to deduce a similar lifetime for these, and hence to take as the upper limit on the excitation rate a value of $10^{-38} \mathrm{sec}^{-1}$.

We wish to illustrate the potential importance of this experimental result for collapse models. Unfortunately, the quarks are moving relativistically, and there are features of the present relativistic collapse models that are not satisfactory. (For more discussion of this see ${ }^{11}$, and references therein). Nevertheless, for purposes of illustration, we will consider a "nonrelativistic proton" and apply SL and CSL to it (a case based on dimensional arguments could be made that a relativistic collapse model would produce similar expressions). First, we consider the prediction of the SL model (eq. (3)), or of the CSL model without any special choice for the coupling constants, eq. (8). Both give an excitation rate

$$
\begin{aligned}
\dot{P}(\phi) & \approx \frac{1}{T}\left(\frac{a_{n u c}}{a}\right)^{2} \\
& \approx \frac{\left(T a^{2}\right)_{G R W}}{\left(T a^{2}\right)} \cdot 10^{-32} \sec ^{-1},
\end{aligned}
$$

leading to the experimental bound

$$
\left(T a^{2}\right)^{-1}<10^{-6}\left(T a^{2}\right)_{G R W}^{-1}=10^{-12} \mathrm{~cm}^{-2} \sec ^{-1},
$$

which is 13 orders of magnitude better than the bound in eq.(4) and is certainly not consistent with the GRW choice of parameters.

Of course the values of $a$ and $T$ used by GRW were only reasonable guesses, and there is some freedom to change them. However, at least in SL, it is very hard to see how they can be changed as much as seems to be required. If, for example, we tried to achieve the bound in eq.(14) by increasing $T$ by a factor $10^{6}$, the collapse time, given in eq.(1), for a pointer containing $10^{23}$ particles would become $10^{-1} \mathrm{sec}$, which is greater than human response times, so that the collapse is failing to do its proper job. On the other hand, suppose we tried to absorb some of the $10^{6}$ factor into a change of $a$, say by increasing $a$ by a factor 10 to one micron, which is greater than a visibly detectable distance. If we consider superposed pointer states with a c.m. separation $l$, on the edge of visibility, so that $\left(\frac{l}{a}\right)^{2}$ is now small compared to one, rather than large as previously assumed, the correct rhs of eq.(1) then has an additional factor $\left(\frac{a}{l}\right)^{2}$, and the collapse time for the pointer is even larger than $10^{-1}$ sec when the limit in eq.(14) is satisfied. It appears therefore, under the assumption 
that the GRW parameters apply to quarks as well as (or instead of) electrons, that the nucleon lifetime experiments can be taken as effectively ruling out the original form of the collapse model.

The CSL model at least partially evades this problem. The extra factor $10^{9}$ noted below eq.(5) allows us to choose $T$ smaller by a similar factor, and therefore well within the bound of eq.(14). Nevertheless, the parameters are uncomfortably close to the bound, especially when we note that proponents of collapse models would want objects much smaller than a $10^{23}$-particle pointer to undergo rapid collapse. For example, a speck of carbon of radius $10^{-3} \mathrm{~cm}$ is visible to the naked eye although it contains only about $10^{15}$ nucleons. If we take a value of $T$ just compatible with eq.(14), i.e., $T=10^{6} T_{G R W}=10^{22} \mathrm{sec}$ then such a speck will collapse (in CSL) in a time of $10^{-2} \mathrm{sec}$, which is on the margin of the perception time. The problem appears even worse if we have to consider experiments where the collapse happens in the brain of an observer. According to the estimates of Aicardi, et al. ${ }^{12}$, an increase of $T$ by a factor of $10^{6}$ would lead to a collapse time of around $1 \mathrm{sec}$ which is unsatisfactorily large.

The situation is much better if the process is such that only the c.m. of the quarks occurs in the lowest-order expansion, and hence in this order there is no excitation. Since the d-quark has about twice the rest mass of the u-quark ( 8 and 4 Mev respectively), this cancellation of the excitation effect in lowest order depends crucially on the couplings being proportional to the mass (see above eq.(9)). (This would not be so crucial in a relativistic model if the effect depended upon energy density, rather than upon mass density as in our discussion, since the energies of the $\mathrm{u}$ and $\mathrm{d}$ quarks are both about $300 \mathrm{Mev}$.) In this case the second-order excitation rate is here down by a factor $10^{-16}$, taking it ten orders of magnitude below that observed, even with the original GRW parameters. Indeed, the model of references 5,6 , based upon a gravitational construction, has precisely this mass-proportional behavior, and similarly avoids experimental refutation. ${ }^{13,14}$

We conclude from this discussion that future experimental results on photon emission from matter applied to present collapse models, and present experimental results on proton decay applied to future relativistic collapse models can have significant consequences for these models: in particular, they can rule out some models and they can indicate that there should be a mass (or energy) proportional coupling, suggestive of a gravitational effect.

\section{Acknowledgment}

We would like to thank GianCarlo Ghirardi, Renata Grassi and Alberto Rimini for helpful comments.

\section{References and Remarks}

1. G.C.Ghirardi and A.Rimini, in Sixty-two years of uncertainty, edited by A.I.Miller (Plenum, New York, 1990) p.167, and G.C.Ghirardi and P.Pearle, in Proc. Philos. of Science Foundation 1990 vol.2, edited by A.Fine, M.Forbes 
and L.Wessels (Phil. Sci Association, Michigan, 1992), p.19 and 35, and further references quoted in these reviews.

2. J.S.Bell in Speakable and unspeakable in quantum mechanics, (Cambridge University Press, Cambridge, 1987), p. 201, and in Sixty-two years of uncertainty, edited by A.I. Miller (Plenum, New York, 1990), p.17, and in Themes in Contemporary Physics II, Essays in honor of Julian Schwinger's 70th birthday, edited by S. Deser and R. J. Finkelstein (World Scientific, Singapore 1989), p. 1.

3. G.C.Ghirardi, A.Rimini and T.Weber, Phys. Rev. D34, 470 (1986).

4. P.Pearle, Phys. Rev. A39, 2277 (1989); G.C.Ghirardi, P.Pearle and A.Rimini, Phys. Rev. A42, 78, (1990).

5. L.Diosi, Phys. Rev. A40, 1165 (1989).

6. G.C.Ghirardi, R.Grassi and A.Rimini, Phys. Rev. A42, 1057, (1990).

7. F.Karolyhazy, Nuovo Cimento 42A, 390 (1966); A. Frenkel, Found. of Phys. 20, 159( 1990); R. Penrose, in Quantum Concepts in Space and Time, edited by R.Penrose and C.Isham (Oxford, 1986); E.J.Squires, The Mystery of the Quantum World (Adam-Hilger, Bristol, 1986) p.111 and 156, and Gravity in configuration space and wavefunction collapse, Durham preprint, 89/15.

8. E.J.Squires, Phys. Letters A158, 432 (1991).

9. This test was suggested to one of us by Tony Leggett: see P.Pearle, Reality Checkpoint, to be published in Acta Enciclopedia (Istituto della Enciclopedia Italiano, 1993).

10. Ch.Berger, et al., (Frejus collaboration), Nuc. Phys. B313, 509 (1989).

11. P.Pearle, in Quantum Chaos - Quantum Measurement, edited by P.Cvitanovic, I.Percival and A.Wirzba (Kluwer, Dordrecht, 1992) p.283.

12. F.Aicardi, A.Borsellino, G.C.Ghirardi and R.Grassi, Found. of Phys. Letters 4, 109 (1991).

13. One attractive feature of the model of references 4,5 is that the constant $T$ is specified in terms of other constants as $\hbar a / G^{2}$. However, this is at least (depending on choice of $m$ ) $10^{7}$ times larger than the $T$ of GRW. This means that the inequality (14) is satisfied even without benefit of the vanishing first order excitation rate. Unfortunately, such a large $T$ makes the collapse time for small objects and brain states rather unacceptably long (see discussion in the two paragraphs following eq.(14)). But, one could give up the $G$ dependence of $T$, keeping the form of the model and increasing $T$ to its GRW value, in which case our discussion of CSL with mass-proportional coupling is valid in all respects for this model too.

14. It also should be mentioned, following a suggestion of Ghirardi and Grassi (private communication), that experimentally satisfactory numbers may also be achieved with a somewhat more complex CSL-type model, in which the reduction rate is proportional to a higher power of the particle number density than the first power (the CSL choice) without having a mass-proportionality or vanishing of the first order excitation rate. With appropriate choices of the power and of a larger value of $T$, one can still achieve a sufficiently short collapse time for even small pointers and for brain states, together with a sufficiently small excitation 
rate to withstand the test of nucleon decay. 